\title{
CONFORMAL MAPS ON HILBERT SPACE
}

\author{
BY MELVYN HUFF
}

Communicated February 15, 1975

1. Introduction. In [1] Nevanlinna gave a simple proof of the following theorem of Liouville. (Precise definitions appear below.)

THEOREM 1. Suppose $U$ is a connected open set in a real Hilbert space $H$ of dimension $\geqslant 3$ (including $\infty$ ) and $f: U \rightarrow H$ is $C^{4}$ and conformal. Then $f$ is either

(a) an affine map whose linear part is a constant multiple of a unitary operator,

(b) an inversion with respect to a sphere,

(c) $f_{1} \circ f_{2}$ where $f_{1}$ is of type (a) and $f_{2}$ is of type (b).

Remarks. (i) The dimension of $H$ must be $\geqslant 3$ because every holomorphic map on $\mathbf{C}$ with a nowhere zero derivative is conformal.

(ii) For $\mathbf{R}^{n}$, the theorem is known even for $f$ just $C^{1}$ [2].

(iii) The proof of Nevanlinna depends on $f$ being $C^{4}$.

In this paper we outline how a technique in [3], when recognized as applying to conformal mappings and suitably modified, can be used to prove the theorem with $f$ only $C^{3}$.

2. Notation and definitions. $H$ will be a real infinite dimensional Hilbert space and $U$ a connected open subset. A map is $C^{n}$ if it is $n$ times continuously Fréchet differentiable as in [4]. A $C^{1}$ function $f: U \rightarrow H$ is called conformal if $D f_{x}$ is a linear isomorphism and there is a function $c: U \rightarrow \mathbf{R}$ such that

$$
\left\langle D f_{x}\left(h_{1}\right), D f_{x}\left(h_{2}\right)\right\rangle=c(x)\left\langle h_{1}, h_{2}\right\rangle
$$

for all $x$ in $U$ and all $h_{1}, h_{2}$ in $H$. (This definition is merely a reformulation of the more geometric definition that says $f$ preserves the angle between two curves meeting at a point.) Banach and Hilbert manifolds are defined as in [4]

By an inversion with respect to the sphere $\{x \in H:\|x-p\|=r\}$ I mean the map $x \rightarrow x^{\prime}$ where

(i) $\|x-p\|\left\|x^{\prime}-p\right\|=r^{2}$ and

(ii) $x$ and $x^{\prime}$ lie on the same ray originating at $p$. The analytic form of such an inversion is

$$
x \rightarrow r^{2}(x-p)\|x-p\|^{-2}+p
$$

AMS (MOS) subject classifications (1970). Primary 58B10, 58B20, 46C10. 
3. Outline of the proof. (1) We develop the theory of connections for Banach manifolds and specialize to the case of Riemannian connections for a $C^{\mathbf{3}}$ Hilbert manifold. For each chart $(W, \psi: W \rightarrow H)$ of the manifold a $C^{1}$ function $\Gamma$, called the Christoffel function, is defined on $\psi(W)$ such that $\Gamma(y)$ is a continuous $H$-valued bilinear map on $H$ for each $y$ in $\psi(W)$. The collection of such $\Gamma$ (together with a coherence property on the overlap of charts) determines and is determined by the connection.

(2) Let $d(x)=1 / \sqrt{c(x)}$. (Since $D f_{x}$ is one-one, $c(x)$ is not zero.) For example if $f$ is the affine map $f(x)=r L_{0}(x)+h_{0}$ where $r$ is real, $L_{0}$ unitary and $h_{0} \in H$, we have $d(x)=1 / r$. On the other hand for the inversion

$$
f(x)=r^{2}(x-p)\|x-p\|^{-2}+p
$$

we have $d(x)=\langle x-p, x-p\rangle / r^{2}$.

Since Hilbert space with the inner product as Riemannian metric has zero curvature we get the following equation for $d$ :

$$
2 D^{2} d_{x}\left(h_{1}, h_{2}\right)=2 D d_{x}\left(h_{1}\right) D d_{x}\left(h_{2}\right) / d(x)+D d_{x}\left[\Gamma_{x}\left(h_{1}, h_{2}\right)\right] \text {. }
$$

To derive this we use the fact that the dimension of $H$ is $\geqslant 3$.

(3) We prove that in a neighborhood of each point $x_{0}$, the above equation has a unique solution

$$
d(x)=A\left\langle x-x_{0}, x-x_{0}\right\rangle+\left\langle b, x-x_{0}\right\rangle+C
$$

where $C=d\left(x_{0}\right), b$ is the element in $H$ corresponding to $D d_{x_{0}}$ under the canonical isomorphism of $H$ with its dual $H^{*}$ and $A=\langle b, b\rangle / 4 C$.

The method of proof is to start at $x_{0}$ where $(* *)$ is true and then to show that equality continues as we move in any direction. Pick a unit vector $u$ and define $g_{1}(t)=d\left(x_{0}+t u\right)$. Using $(*)$ the function $K_{1}(t)=\left[g_{1}(t), D g_{1}(t)\right] \in R$ $\times H^{*}$ is shown to satisfy a differential equation of the form $K^{\prime}(t)=F[t, K(t)]$ with initial condition $K(0)=\left[d\left(x_{0}\right), D d_{x_{0}}\right]$. Letting $g_{2}(t)=A\langle t u, t u\rangle+\langle b, t u\rangle$ $+C$ we verify that $K_{2}(t)=\left[g_{2}(t), D g_{2}(t)\right]$ satisfies the same differential equation and initial condition. The equality of $g_{1}$ and $g_{2}$ follows from uniqueness.

(4) Using the connectedness of $U$ we get that the local solution in (3) is actually a global solution for $d$.

(5) We show that if $f: U \rightarrow H$ and $g: U \rightarrow H$ are $C^{3}$ conformal maps such that $g$ is one-one and $d_{f}=d_{g}$ (where $d_{f}$ is the $d$ corresponding to $f$ ), then there is a vector $h$ in $H$ and unitary operator $L$ such that $f=L \circ g+h$.

(6) From (4) we know that $d(x)=A\left\langle x-x_{0}, x-x_{0}\right\rangle+\left\langle b, x-x_{0}\right\rangle+C$.

Case 1. $b=0$ and $A=\langle b, b\rangle / 4 C=0$ in which case $d(x)=C$ has the same form as the $d$ for an affine map as in (2), if $C=1 / r$.

Case 2. $b \neq 0$ and thus $A \neq 0$. Then $d(x)=\left\langle x-p_{0}, x-p_{0}\right\rangle / r$ where $r=$ $4 C /\langle b, b\rangle$ and $p=x_{0}+2 C b /\langle b, b\rangle$. This is the same form as the $d$ for an inversion as in (2) above. 
(7) Combining (6) with (5) we get our theorem.

\section{REFERENCES}

1. R. Nevanlinna, On differentiable mappings, Analytic Functions, Princeton Univ. Press, Princeton, N. J., 1960, pp. 3-9. MR 22 \#7075.

2. Ju. G. Rešetnjak, On conformal mappings of a space, Dokl. Akad. Nauk SSSR 130 (1960), 1196-1198 = Soviet Math. Dokl. 1 (1960), 153-155. MR 22 \#9935.

3. L. Eisenhart, Riemannian geometry, Princeton Univ. Press, Princeton, N. J. 1960, p. 85 .

4. S. Lang, Introduction to differentiable manifolds, Interscience, New York, 1962. MR 27 \#5192.

DEPARTMENT OF MATHEMATICS, TUFTS UNIVERSITY, MEDFORD, MASSACHUSETTS 02155

Current address: 329A Summit Ave., Brookline, Massachusetts 